\title{
REFRIGERAÇÃO UTILIZANDO PASTILHAS DE EFEITO PELTIER
}

\author{
Jainne Daniele F. S. Fernandes \\ Alunos do Curso Técnico em Eletrotécnica do Campus Natal - Zona Norte do IFRN. \\ Alailson de Freitas Braz \\ Alunos do Curso Técnico em Eletrotécnica do Campus Natal - Zona Norte do IFRN. \\ Ludmila Sayonara S. X. Machado \\ Alunos do Curso Técnico em Eletrotécnica do Campus Natal - Zona Norte do IFRN. \\ Edfranklin de Souza Pimentel \\ Alunos do Curso Técnico em Eletrotécnica do Campus Natal - Zona Norte do IFRN. \\ Aécio Vinícius Amorim Farias \\ ${ }^{1}$ Técnico de Laboratório do IFRN. E-mail: aecio@cefetrn.br. \\ Antonio Luiz Pereira de Siqueira Campos \\ Professor da UFRN. E-mail: antonio.luiz@pq.cnpq.br.
}

\section{RESUMO}

Pastilhas de Peltier são dispositivos baseados no efeito Peltier e são aplicadas para produzir efeito refrigerador ou aquecedor. Este trabalho aborda conceitos envolvidos na fabricação e funcionamento desses dispositivos tais como: histórico do efeito Peltier e desenvolvimento de placas de efeito Peltier. É feito um estudo de eficiência da refrigeração de água utilizando uma placa de efeito Peltier existente no Campus Natal - Zona Norte. Para isso, é implementada uma estrutura de refrigeração de água e são feitas medições para determinar a eficiência de sistema. Por fim, são apresentadas conclusões sobre o estudo desenvolvido.

PALAVRAS-CHAVE: Efeito Peltier, Placas de efeito Peltier, Refrigeração.

\section{REFRIGERATION USING PELTIER EFFECT WALFERS}

\begin{abstract}
Peltier's wafers are devices based on Peltier effect and it is applied to produce a cooler or heater effect. This paper points to concepts involved in the manufacture and operation of these devices such as: history of the Peltier effect and development of Peltier wafers. It is shown the efficiency of cooling water using a Peltier wafer existing in Campus Natal Zona Norte. For this, we implemented a set of cooling water and measurements were made to determine the efficiency of system. Finally, conclusions are presented about the measurements realized.
\end{abstract}

KEYWORDS: Peltier effect, Peltier wafer, Refrigeration. 


\section{REFRIGERAÇÃO UTILIZANDO PASTILHAS DE EFEITO PELTIER}

\section{INTRODUÇÃO}

Em 1834, o físico francês Jean Charles Athanase Peltier observou que uma junção de dois metais, bismuto e cobre ( $\mathrm{Bi}$ e $\mathrm{Cu}$ ) se tornava mais quente quando era percorrida por uma corrente elétrica no sentido do bismuto para o cobre e esfriava, quando esse sentido era invertido, constituindo-se, portanto, em um efeito eletrotérmico denominado mais tarde de efeito Peltier.

Em 1827 e em 1828, o físico italiano Leopoldo Nobili realizou dois tipos de experiências nas quais observou a relação entre corrente elétrica e gradiente de temperatura. Na primeira delas, ele observou o que ocorre quando há um gradiente de temperatura através do condutor no qual circula uma corrente elétrica, denominada por ele de corrente termoelétrica; na segunda, ele estudou a corrente elétrica que é gerada em processos envolvendo condutores úmidos, como a corrente voltaica, denominada por ele de corrente hidroelétrica. Observe-se que, em 1835, Nobili voltou a realizar novas experiências sobre o efeito eletrotérmico.

Estimulado pelo trabalho de Nobili, Peltier construiu um galvanômetro sensível para medir a condutividade do antimônio $(\mathrm{Sb})$ e do bismuto $(\mathrm{Bi})$ para pequenas correntes elétricas. $\mathrm{O}$ comportamento térmico anômalo apresentado por esses materiais levou-o a construir um termoscópio termoelétrico e a medir a distribuição da temperatura em um termopar (Bi$\mathrm{Cu}$ ). Substituindo o termoscópio por um termômetro de ar, Peltier fez então a descoberta do efeito peltier.

É oportuno destacar que, em suas experiências iniciais com o Sb e o Bi, Peltier observou que a corrente elétrica produzia uma elevação de temperatura em todas as partes desses condutores, desde que estes mantivessem o mesmo diâmetro. Contudo, como ele estava preocupado apenas com a elevação de temperatura e não com a quantidade de calor envolvida no processo, não chegou a relacionar esta com a intensidade da corrente elétrica.

Esta relação só seria encontrada em 1841 pelo físico inglês James Prescott Joule. Ele tomou um fio metálico e ligou-o a uma pilha de Volta. Mediu então a quantidade de calor por unidade de tempo dissipada no fio devido à corrente elétrica gerada pela pilha. Em decorrência disso, encontrou, então, que a quantidade de calor era proporcional à resistência elétrica do fio multiplicado pelo quadrado da corrente elétrica.

O efeito Peltier é a produção de um gradiente de temperatura entre duas junções de dois condutores (ou semicondutores) de materiais diferentes quando submetidos a uma diferença de potencial elétrica em um circuito fechado.

\section{PLACAS DE EFEITO PELTIER}

Placas de efeito Peltier, também conhecidas como pastilhas termoelétricas utilizam o efeito refrigerador ou aquecedor ao se fazer passar corrente elétrica contínua por dois condutores. Com uma voltagem aplicada entre os pólos, cria-se um diferencial de temperatura entre as faces opostas da placa. Basicamente, as pastilhas são formadas por semicondutores do tipo P e tipo N. Esses elementos semicondutores são soldados entre duas placas cerâmicas, eletricamente em série e termicamente em paralelo. Essa placa é ilustrada na Figura 1. 


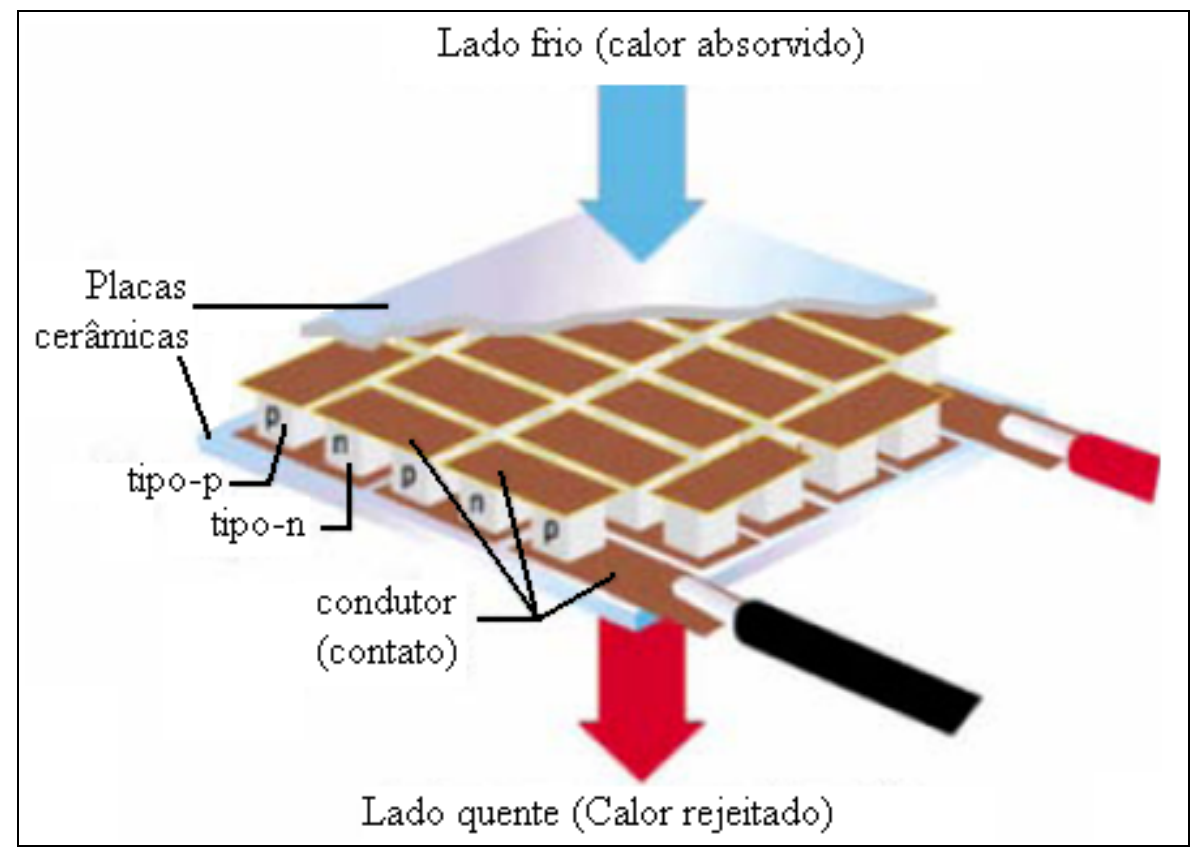

Figura 1 - Pastilha termoelétrica.

Para se evitar superaquecimento das placas, o uso de dissipadores de calor e ventiladores é obrigatório tanto do lado quente quanto do lado frio. Para a montagem, recomenda-se o uso de pasta térmica entre a placa e o dissipador, para que se aumente a eficiência de troca térmica. Esse esquema é ilustrado na Figura 2.

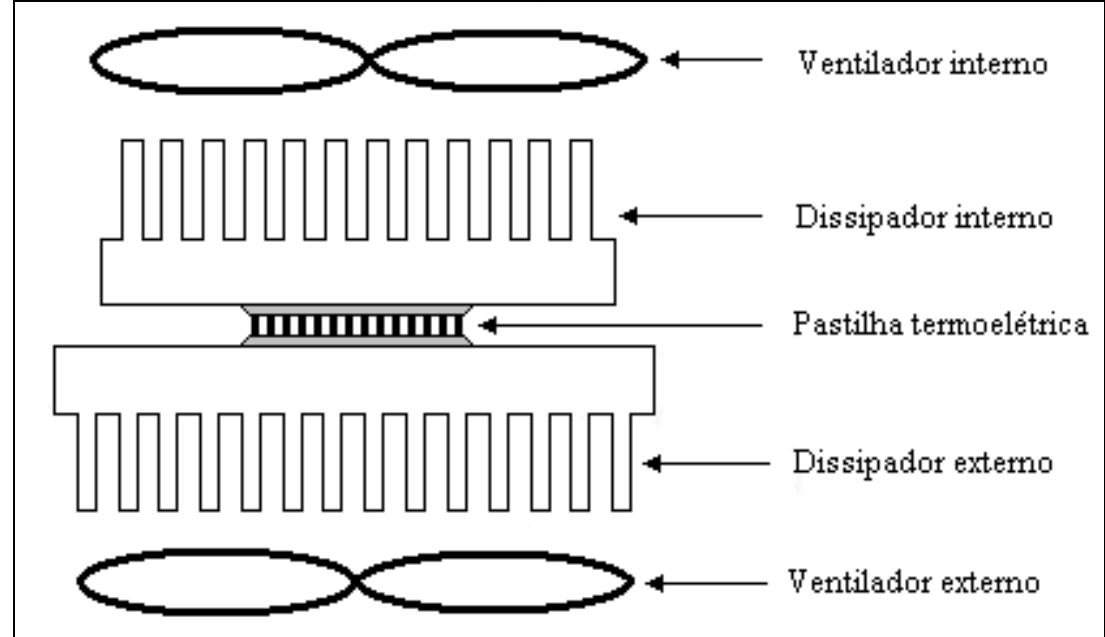

Figura 2 - Configuração típica para montagem da pastilha termoelétrica.

Entre as vantagens do uso dos módulos Peltier, podemos destacar: é extremamente leve, possui controle de temperatura preciso, é totalmente silencioso, não há vibração, requer menos espaço físico que os ciclos por compressão e absorção e requer menos manutenção.

Vale ressaltar que cada pastilha tem seu próprio limite da quantidade de calor que ela pode transferir, conhecido como Qmax. A corrente elétrica associada ao Qmax é conhecida como Imax, e a tensão elétrica correspondente como Vmax.

A eficiência da conversão da energia, ou coeficiente de desempenho (COP) de dispositivos termoelétricos refrigeradores, é determinada pela figura de mérito termoelétrica, 
comumente denotada por ZT. Refrigeradores baseados em tais materiais, tipicamente, têm um COP de valor, aproximadamente, igual a 2, que é mais baixa que o COP de 3 a 4 do refrigeradores de compressão de vapor. Entretanto, não há impedimento teórico conhecido para aumentar significativamente a eficiência da conversão da energia termoelétrica.

\section{RESULTADOS}

Foi montado um sistema com uma cuba de água, dois multímetros e uma estrutura para se medir a variação da temperatura da água e do dissipador de calor. Esse conjunto de medições está ilustrado na foto da Figura 3.

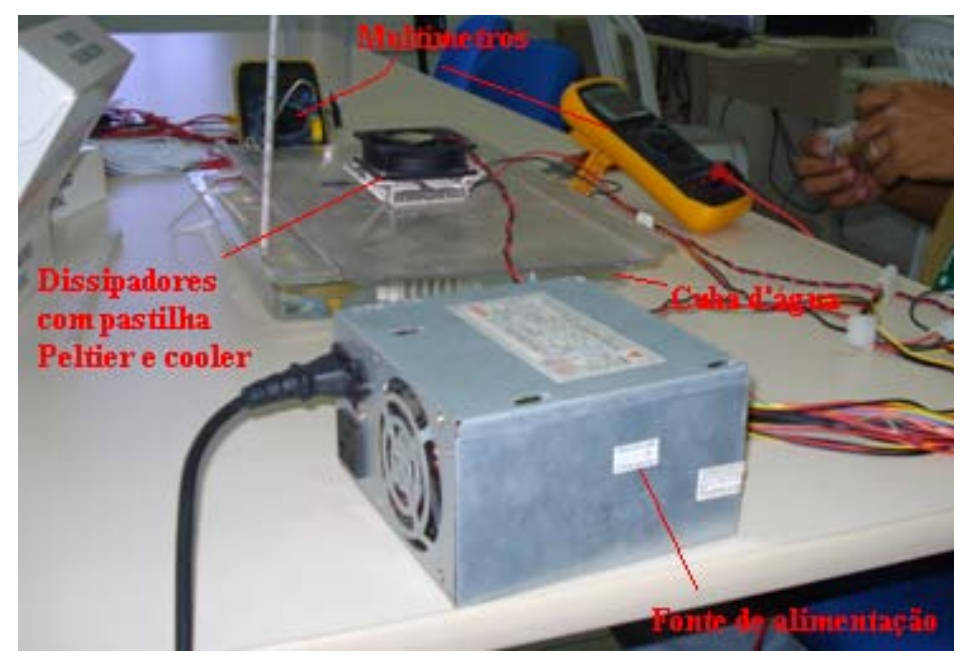

Figura 3 - Sistema de refrigeração d’água montado.

A foto da Figura 4 ilustra detalhes dos dissipadores. Entre eles está a pastilha de Peltier. Um dissipador é resfriado pela água e o outro é resfriado pelo cooler.

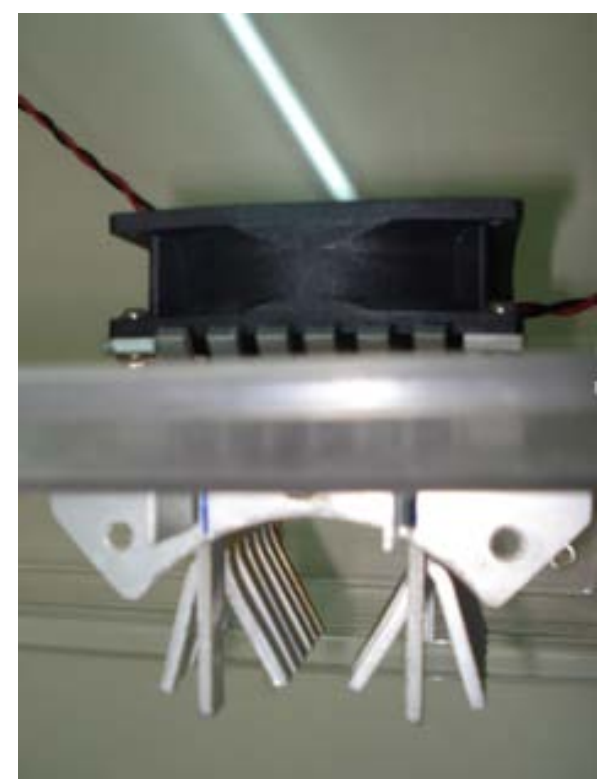

Figura 4 - Detalhe dos dissipadores utilizados no sistema. 
A pastilha de Peltier utilizada no sistema é mostrada na Figura 5. Ela é uma pastilha de aproximadamente $4 \mathrm{~W}$ e foi realizada a medição do seu consumo. A tensão medida foi de $3,38 \mathrm{~V}$ e a corrente medida foi de $1,07 \mathrm{~A}$.

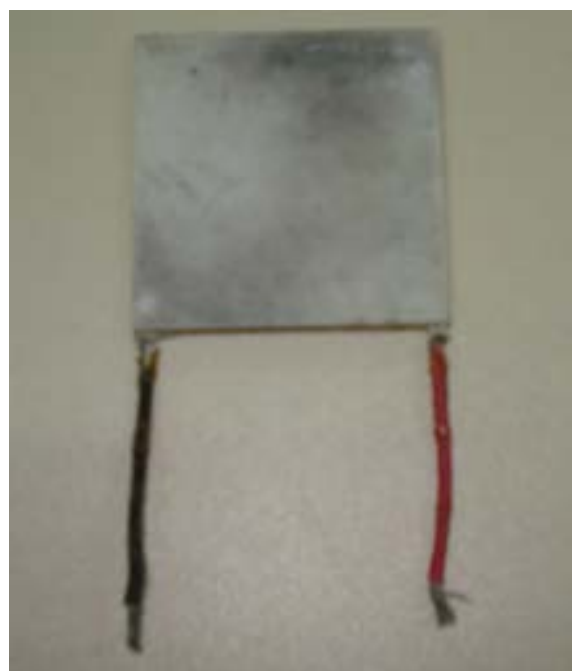

Figura 5 - Pastilha de Peltier de 4 W.

Com o aparato montado, foi medida a variação da temperatura em função do tempo. A Figura 6 mostra essa variação. Pode-se observar que, em uma hora, a água foi resfriada em $5{ }^{\circ} \mathrm{C}$, havendo um consumo de $4 \mathrm{Wh}$.

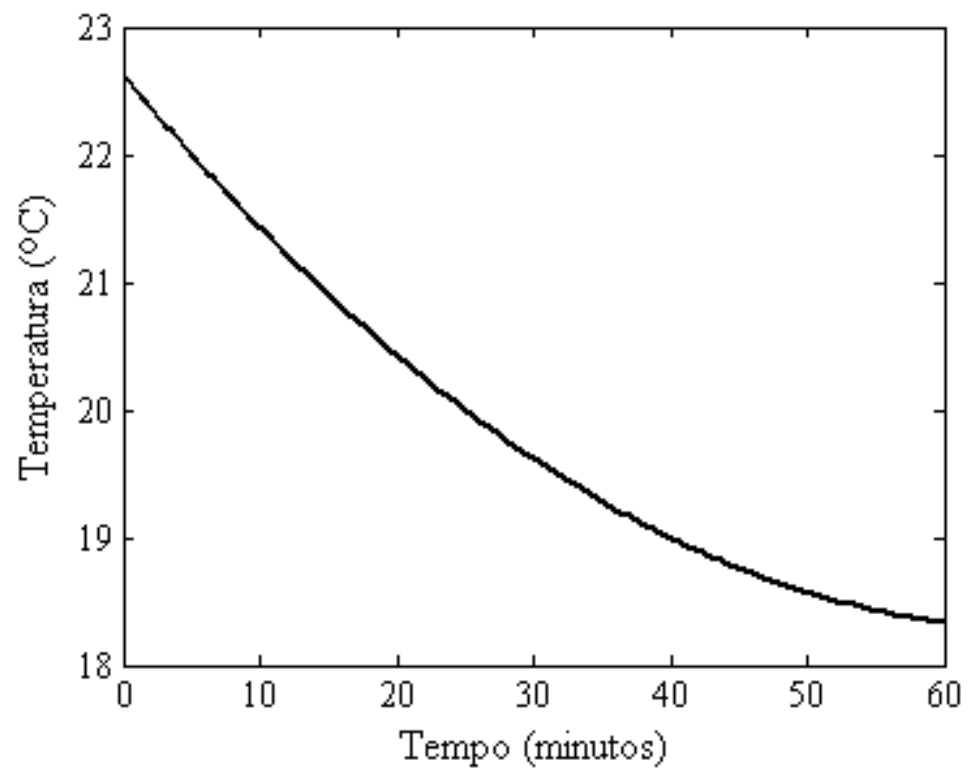

Figura 6 - Curva da temperatura da água em função do tempo.

\section{APLICAÇÕES E PESQUISAS}

Existem inúmeros produtos no mercado que utilizam pastilhas de Peltier. A Iceberg, fabricante de coolers para processadores de computadores, lançou uma linha de coolers que utilizam pastilhas de Peltier. Um desses tipos de coolers pode ser visto na foto da Figura 7 (PETER, 2010). 


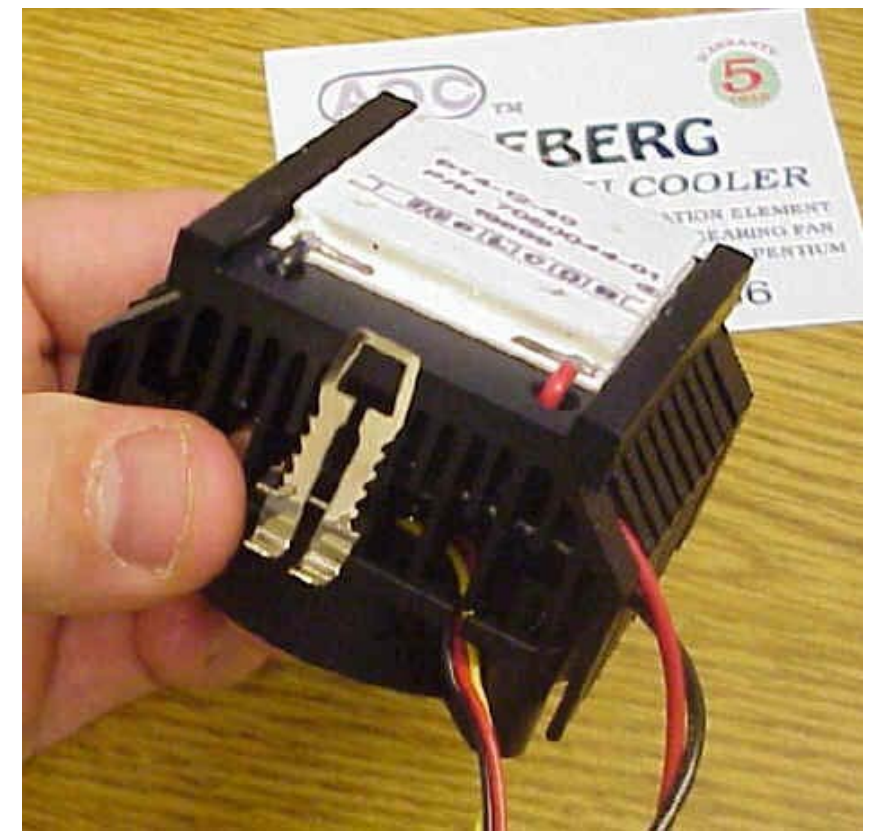

Figura 7 - Cooler que utiliza pastilha de Peltier (PETER, 2010).

Outra aplicação comum para pastilhas de Peltier é em mini-refrigeradores. Existem inúmeros fabricantes e modelos no mercado. A Figura 8 ilustra alguns exemplos de minirefrigeradores.

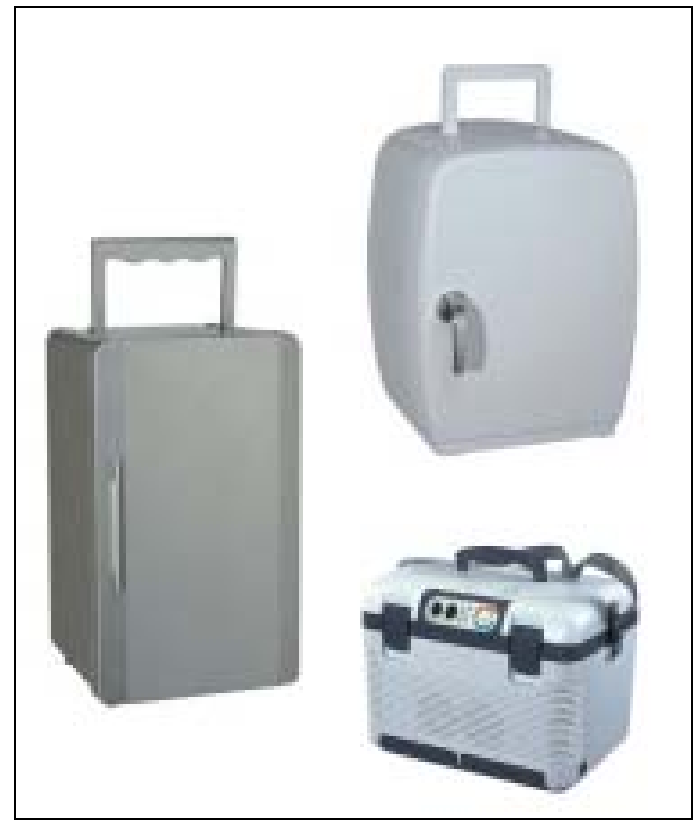

Figura 8 - Mini-refrigeradores que utilizam pastilhas de Peltier.

A FIAT cogita lançar uma linha de condicionadores de ar automotivos que também utilizariam essa tecnologia. Entretanto, os produtos mais comuns que utilizam pastilhas de Peltier são mesmo os pequenos refrigeradores.

No campo das pesquisas, existem inúmeros estudos para desenvolver aplicações comerciais que utilizam pastilhas Peltier. Tan et al. Desenvolveu uma pesquisa para desenolver um resfriador termoelétrico (TEC) que é uma bomba de calor de estado sólido, que utiliza pastilhas Peltier, para integração de dispositivos (TAN, 2005). No Rio Grande 
do Norte, podemos citar o professor Feitosa da UFRN, que desenvolveu inúmeras pesquisas envolvendo pastilhas de Peltier. NISHIHATA et al. relataram em (NISHIHATA, 2002) o desenvolvimento de um trocador de calor líquido de alta eficiência e elevada capacidade no qual um módulo termoelétrico foi instalado para minimizar o consumo de energia elétrica e reduzir os custos. Por fim, podemos relatar a pesquisa desenvolvida na Universidade de São Paulo, na qual alunos do curso de engenharia elétrica desenvolveram uma micro-adega climatizada de baixo custo utilizando pastilhas de Peltier (STRAZZA, 2004). Estes são apenas alguns exemplos, dentre os inúmeros que nós podemos encontrar na literatura.

\section{CONCLUSÕES}

Este trabalho abordou conceitos envolvidos na fabricação e funcionamento de pastilhas de Peltier. Foi feito um estudo de eficiência da refrigeração de água utilizando uma placa de efeito Peltier existente no Campus Natal - Zona Norte. Para isso, foi implementada uma estrutura de refrigeração de água e foram feitas medições para determinar a eficiência do sistema. O experimento demonstrou a viabilidade do uso de pastilhas de Peltier em pequenos dispositivos refrigerados como bebedouros e pequenas geladeiras. Para isso, basta aumentar o número e a potência das pastilhas de Peltier.

\section{REFERÊNCIAS BIBLIOGRÁFICAS}

1. — Efeitos: Seebeck, Peltier, Joule e Thomson. Disponível em: http://www.bassalo.com.br/cf10_curiosidade198.pdf. Acessado em outubro de 2009.

2. — Thermo Generator - Peltier Effect. Disponível em: http://www.scientrific.com.au/PDFs/hl4052-001.pdf. Acessado em outubro de 2009.

3. DUARTE, G. F. R. e CARLSON, B. V., Geradores Termoelétricos Radioisotópicos. Disponível em: http://www.bibl.ita.br/xiencita/Artigos/Fund15.pdf. Acessado em outubro de 2009.

4. FEITOSA, C. C. A., Dispositivos Peltiers como Mini-refrigeradores, Currículo Lattes. Disponível em: http://www.cnpq.br. Acessado em outubro de 2009.

5. PADDOCK, B., A Guide to online information about: Peltier Thermoelectric Coolers. Disponível em: http://www.designer-iii.com/cco/Peltier.pdf. Acessado em outubro de 2009.

6. PETER, K., The Peltier Cooled PC: Considerations for Power Consumption and Heat. Disponível em: http://www.thetechzone.com/articles/overclocking/peltier_pc/. Acessado em 11/06/2010.

7. NISHIHATA, H., KIDO, O. e UENO, T., Peltier Cooling System Utilizing Liquid Heat Exchanger Combined with Pump, 21st International Conference on Thermoelectronics, Estados Unidos, 2002.

8. STRAZZA, F. e RIBERI, R. M., Projeto de uma Micro-Adega Climatizada de Baixo Custo. Monografia de Final de Curso. Universidade de São Paulo - USP, 2004.

9. TAN, Y. M. et al., Fabrication of Thermoelectric Cooler for Device Integration, 2005 Electronics Packaging Technology Conference, China, 2005. 